\section{The use of mean platelet volume for distinguishing the causes of thrombocytopenia in adult patients}

\author{
Lalita Norrasethada, ${ }^{1}$ \\ Wichan Khumpoo, ${ }^{2}$ \\ Ekarat Rattarittamrong, ${ }^{1}$ \\ Thanawat Rattanathammethee, ${ }^{1}$ \\ Chatree Chai-Adisaksopha, ${ }^{1}$ \\ Adisak Tantiworawit ${ }^{1}$
}

${ }^{1}$ Division of Hematology, Department of Internal Medicine, and ${ }^{2}$ Department of Internal Medicine, Faculty of Medicine, Chiang Mai University, Thailand

\begin{abstract}
Establishment and analysis of mean platelet volume (MPV) may be helpful in the discrimination between underproduction or over-destruction of platelets as the causes of thrombocytopenia. The primary objective is to find the cut-off point of MPV for distinguishing causes of thrombocytopenia. The secondary objective is to validate the cut-off value of the MPV by using bone marrow examination. Thrombocytopenic patients were enrolled in a training set and a receiving operating characteristics (ROC) curve was plotted to obtain the cut-off value of MPV. A validation set of patients was recruited to validate the cut-off value. The training set included 240 patients. Half with with underproductive $(n=120)$ and half with over-destructive thrombocytopenia $(\mathrm{n}=120)$. The best cut-off value of MPV was $8.8 \mathrm{fL}$. The validation set included 119 patients in total, again in 2 groups, those with underproductive $(n=84)$ and those with overdestructive thrombocytopenia $(n=35)$. The sensitivity, specificity, PPV and NPV when $\mathrm{MPV} \geq 8.8 \mathrm{fL}$ indicating over-destructive thrombocytopenia were $77 \%, 89 \%, 89 \%$ and $77 \%$, respectively. MPV is useful for differentiating the cause of thrombocytopenia. The value of MPV $\geq 8.8 \mathrm{fL}$ has acceptable sensitivity and specificity for diagnosis of over-destructive thrombocytopenia.
\end{abstract}

\section{Introduction}

Platelets are the major blood component involved in primary hemostasis. Thrombocytopenia or a decrease in the level of platelets to less than $150 \times 10^{9} / \mathrm{L}$ may lead to bleeding complications. The causes of thrombocytopenia can be divided into two major groups, a decreased production of platelets or an underproliferative bone marrow (BM) and an over-destruction of platelets. ${ }^{1}$ Underproliferative BM defects include aplastic anemia (AA), acute leukemia, myelodysplastic syndrome (MDS), and chemotherapy-induced thrombocytopenia. On the contrary, peripheral destruction of platelets may be as a result of an immune-mediated process such as immune thrombocytopenia (ITP) or a nonimmune mediated one including disseminated intravascular coagulopathy (DIC), thrombotic thrombocytopenic purpura (TTP) or certain infections. ${ }^{1}$

A BM examination is considered as being a standard procedure in differentiating between these two pathogeneses of thrombocytopenia if the etiology is unclear. ${ }^{1}$ This procedure is important for the diagnosis of underproductive BM disorders. However, it is an invasive procedure and may be not necessary for establishing a clear diagnosis of peripheral destruction disorders such as ITP 2 and DIC. ${ }^{3}$

Currently, automated blood analyzers are being developed and have many parameters which can be used to identify the causes of thrombocytopenia. Mean platelet volume (MPV) is one of automated platelet indices that has been investigated by many studies around the achievement of this objective due to its widespread availability. ${ }^{4}$ Previous earlier studies showed that patients with BM hypoplasia or thrombocytopenia resulting from cytotoxic drugs or chemotherapy had a low MPV. ${ }^{5-7}$ In contrast, disorders involving the peripheral destruction of platelets had a higher MPV compared to BM diseases. ${ }^{7}$ As a result, MPV may be the best non-invasive tool to use to differentiate between the two main pathogeneses of thrombocytopenia. However, the cut-off value, inclusion and exclusion criteria differed between trials, along with a lack of validation limit in the use of MPV in clinical practice. ${ }^{4}$

The primary objective of this study is to find the cut-off point of MPV for distinguishing the causes of thrombocytopenia. The secondary objective is to validate the cut-off value of MPV when using BM examination as a gold standard test.

\section{Materials and Methods}

\section{Study overview}

This was a cross-sectional study conducted at Chiang Mai University Hospital. The local Institutional Review Board approved the study stating it was in accordance with the guidelines given in the Declaration of Helsinki. Two cohorts of thrombocytopenic patients were enrolled
Correspondence: Ekarat Rattarittamrong, Division of Hematology, Department of Internal Medicine,

Faculty of Medicine, Chiang Mai University, Chiang Mai 50200, Thailand.

Tel: +66819296335 - Fax: +6653935481.

E-mail: ekarat.r@cmu.ac.th

Key words: Thrombocytopenia; mean platelet volume; underproductive thrombocytopenia; over-destructive thrombocytopenia.

Contributions: LN designed the research, carried out data analysis, and co-wrote the manuscript; WK obtained the research grant, collected and summarized the clinical data, carried out the data analysis, and co-wrote the manuscript; ER co-wrote and revised the manuscript; TR, CC, AT revised the manuscript.

Conflict of interest: the authors declare no potential conflict of interest.

Funding: this research had funding support from the Faculty of Medicine, Chiang Mai University, Thailand.

Received for publication: 25 April 2018.

Revision received: 11 January 2019.

Accepted for publication: 25 March 2019.

This work is licensed under a Creative Commons Attribution-NonCommercial 4.0 International License (CC BY-NC 4.0).

(C) Copyright L. Norrasethada et al., 2019

Licensee PAGEPress, Italy

Hematology Reports 2019; 11:7732

doi:10.4081/hr.2019.7732

onto the study, a training set and a validation set. The training set included patients who were admitted to hospital or attended out-patient clinics between $1^{\text {st }}$ June 2013 and $31^{\text {st }}$ May 2014. The data from this cohort was collected and analyzed from their medical records. The validation set included patients who were admitted or attended during the period $1^{\text {st }}$ June 2014 to $31^{\text {st }}$ June 2015.

The objective with the training set was to find a cut-off value of MPV that has appropriate sensitivity and specificity to differentiate between underproliferative and over-destructive causes of thrombocytopenia. The inclusion criteria were patients with thrombocytopenia with platelets $<100 \times 10^{9} / \mathrm{L}$ who had MPV data. The diagnoses were established by hematologists and internists. The number of patients needed to ensure the study was statistically valid was calculated using the formula: $\mathrm{N}=\mathrm{Z}^{2}$ [sensitivity (1-sensitivity)] $/ \mathrm{e}^{2} \quad$ where $\mathrm{Z}=95 \%$ confidence interval $(95 \% \mathrm{CI})$ which was 1.96 , sensitivity $=0.8$, and $\mathrm{e}=$ error was set at 0.05 . Using this, the population in 
the training set needed to be 240 with 120 patients being identified as those with underproduction and 120 patients with peripheral destruction as regards causes of thrombocytopenia.

The validation set aimed to validate the cut-off value of MPV derived from the training set. The inclusion criteria were thrombocytopenic patients (platelets $<100 \times 10^{9} / \mathrm{L}$ ) who had undergone a BM study which had been interpreted by hematologists. Exclusion criteria were patients who had received a platelet transfusion prior to MPV analysis.

Data pertinent to clinical characteristics including age, gender, and diagnosis as well as $\mathrm{CBC}$ and MPV results were collected from both cohorts. CBC and MPV were derived using an automated blood analyzer (Beckman Coulter LH780).

\section{Statistical analysis}

Clinical characteristics of patients and $\mathrm{CBC}$ results in both cohorts were analyzed as descriptive methods including mean \pm standard deviation (SD). Chi-square test, student t-test, and Wilcoxon rank sum test were used to compare clinical characteristics and $\mathrm{CBC}$ between patients with underproductive $\mathrm{BM}$ disorders and over-destruction disorders depending on type and distribution of variables. A P-value of less than 0.05 was used to determine statistical significance. The sensitivity and specificity of each cutoff value of MPV were analyzed from the training set. A receiving operating characteristics (ROC) curve was subsequently plotted to obtain the cutoff value of MPV. The sensitivity, specificity, positive predictive value (PPV), negative predictive value (NPV), and accuracy of the cut-off value of MPV derived from the training set were re-analyzed in the validation cohort. SPSS version 16.0 was used to analyze the data.

\section{Results}

\section{Training set}

There were 240 patients in the training set with 120 patients in the underproductive BM group and 120 patients in the overdestruction group as shown in Table 1. The majority of patients in the underproductive $\mathrm{BM}$ group were diagnosed with AML and AA whereas more than half the patients in the over-destruction group had DIC and about one-third suffered from ITP. The underproductive $\mathrm{BM}$ group had a predominance of females $(60 \%$ vs. $47 \%, \mathrm{P}=0.03)$ and was overall of younger age than the over-destruction group (mean age, 50.3 years vs. 55.9 years, $\mathrm{P}=0.01$ ). The patients with underproductive $\mathrm{BM}$ also had lower $\mathrm{Hb}$ levels compared to the over-destruction group (mean $\pm \mathrm{SD}, 8.3 \pm 1.6 \mathrm{~g} / \mathrm{dL} v s .9 .6 \pm 2.5$ $\mathrm{g} / \mathrm{dL}, \mathrm{P}=0.005$ ). The MPV was significantly higher in the over-destruction group (mean $\pm \mathrm{SD}, 9.6 \pm 2.4$ vs. $9.0 \pm 1.8 \mathrm{fL}, \mathrm{P}=0.02$ ).

The ROC curve obtained from the MPV of patients in the training set had an area under the curve of 0.57 (Figure 1). The sen- sitivity MPV of 8.0, 8.8, and 9.0 fL in the differentiation between underproductive bone marrow and over-destruction causes of thrombocytopenia was $75 \%, 60 \%$, and $50 \%$, respectively whereas the specificity values of each cut-off were $30 \%, 50 \%$, and $54 \%$, respectively. Therefore, the cut-off of MPV at $8.8 \mathrm{fL}$ that had a sensitivity of $60 \%$ and specificity of $50 \%$ was tested in the validation cohort.

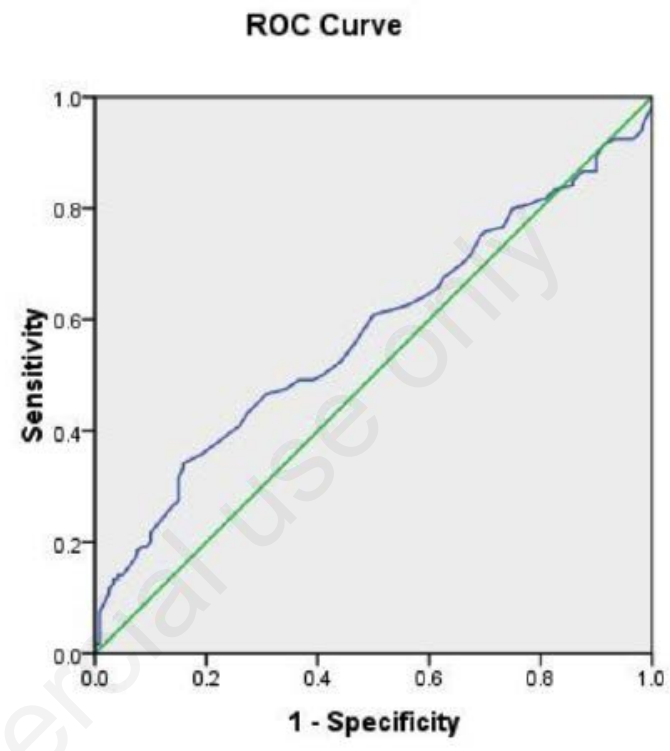

Diagonal segments are produced by ties.

Figure 1. Receiving Operating Characteristics curve of mean platelet volume to differentiate between underproductive bone marrow and over-destruction causes of thrombocytopenia.

Table 1. Comparison of clinical characteristics between patients with underproductive bone marrow and those with over-destruction of platelets in the training set.

\begin{tabular}{|c|c|c|c|}
\hline Parameter & $\begin{array}{l}\text { Underproductive } \\
\text { bone marrow, } \\
\text { N=120 (\%) }\end{array}$ & $\begin{array}{c}\text { Over-destruction } \\
\text { of platelets, } \\
\mathrm{N}=120(\%)\end{array}$ & P-value \\
\hline $\begin{array}{l}\text { Gender } \\
\text { Male } \\
\text { Female }\end{array}$ & $\begin{array}{l}48(40) \\
72(60)\end{array}$ & $\begin{array}{l}64(53) \\
56(47)\end{array}$ & 0.03 \\
\hline Age (years), Mean \pm SD & $50.3 \pm 18.4$ & $55.9 \pm 15.6$ & 0.01 \\
\hline Diagnosis (\%) & $\begin{array}{c}\text { AA, } 38(31.6) \\
\text { AML, } 53(44.2) \\
\text { ALL, } 12(10.0) \\
\text { CMT, } 17(14.2)\end{array}$ & $\begin{array}{c}\text { ITP, } 40(33.3) \\
\text { DIC, } 65(54.2) \\
\text { TTP, } 4(3.3) \\
\text { DHF, } 11(9.2)\end{array}$ & - \\
\hline Hemoglobin (g/dL), Mean \pm SD & $8.3 \pm 1.6$ & $9.6 \pm 2.5$ & 0.005 \\
\hline White blood count $\left(\times 10^{9} / \mathrm{L}\right)$, Mean \pm SD & $4.5 \pm 145.8$ & $6.6 \pm 8.2$ & 0.46 \\
\hline Platelet count $\left(\times 10^{9} / \mathrm{L}\right)$, Mean \pm SD & $33.5 \pm 27.2$ & $38.2 \pm 24.3$ & 0.37 \\
\hline Mean platelet volume (fL), Mean \pm SD & $9.0 \pm 1.8$ & $9.6 \pm 2.4$ & 0.02 \\
\hline
\end{tabular}

AA, aplastic anemia; AML, acute myeloid leukemia (pre-chemotherapy); ALL, acute lymphoblastic leukemia (pre-chemotherapy); CMT, chemotherapy-induced myelosuppression; ITP, immune thrombocytopenia; DIC, disseminated intravascular coagulation; TTP, thrombotic thrombocytopenia purpura; DHF, dengue hemorrhagic fever. 


\section{Validation set}

One hundred and nineteen patients who had undergone BM investigation were included in the validation set. Eighty-four patients were in the underproductive BM group and 35 patients in the over-destruction group (Table 2). Similar to the findings from the training set, most patients in the underproductive BM group were diagnosed with AML and AA. However, all patients in the over-destruction group had ITP. In both groups, males predominated with a mean age \pm SD of $43.5 \pm 18.4$ and $50.0 \pm 20.0$ years, respectively. The patients with underproductive $\mathrm{BM}$ had lower $\mathrm{Hb}$ (mean $\pm \mathrm{SD}$, $8.4 \pm 1.8 \mathrm{~g} / \mathrm{dL} v s .9 .9 \pm 2.5 \mathrm{~g} / \mathrm{dL}, \mathrm{P}=0.03)$ and lower WBC counts (mean $\pm \mathrm{SD}, 2.6 \pm 36$ $\times 10^{9} / \mathrm{L}$ vs. $\left.6.7 \pm 6.3 \times 10^{9} / \mathrm{L}, \mathrm{P}=0.03\right)$ compared to those recorded in the over-destruction group. The over-destruction group had a higher MPV (mean \pm SD, 10.4 $\pm 2.1 v s$. $7.2 \pm 1.2 \mathrm{fL}, \mathrm{P}=0.03)$. By applying the cutoff value of $8.8 \mathrm{fL}$ in this cohort, the patients can be divided into 4 groups according to causes of thrombocytopenia based on the BM study results and MPV. In the over-destruction of platelet group, 27 out of 35 patients $(77.1 \%)$ had MPV $\geq 8.8 \mathrm{fL}$ whereas only 8 patients out of 84 patients $(9.5 \%)$ from the underproductive bone marrow group had an MPV over the same cutoff value. As a consequence, sensitivity, specificity, PPV, NPV, and accuracy of $\mathrm{MPV} \geq 8.8 \mathrm{fL}$ indicating over-destruction causes of thrombocytopenia were $77 \%$, $89 \%, 89 \%, 77 \%$, and $86 \%$, respectively.

\section{Discussion and Conclusions}

In peripheral destruction causes of thrombocytopenia production of platelets is stimulated to compensate for their destruction and leads to an increase in the immature form of platelets. ${ }^{8}$ Since the size of the immature platelets is larger than the mature form, MPV measured using an automated hematology analyzer in cases of peripheral destruction is usually higher than cases involving BM defects. ${ }^{7}$ Analytical findings of the data from both the training cohort and validation cohort from this study supported this hypothesis. In the validation cohort, where the causes of thrombocytopenia were confirmed by BM studies, the mean MPV in the over-destruction group was $10.4 \mathrm{fL}$ compared to $7.2 \mathrm{fL}$ in the underproductive $\mathrm{BM}$ group. The mean MPV in the overdestruction group was close to a previous study $(9.8 \mathrm{fL})^{7}$ as well as those found in other studies into ITP patients (9.86-12.4 fL). ${ }^{9-11}$ To the contrary, MPV was demonstrated to be lower in patients with under- productive BM defects, a finding similar to the data reported in previous studies (6.2$8.1 \mathrm{fL}){ }^{7,12}$ When using a cut-off value of $8.8 \mathrm{fL}$, the sensitivity, specificity, PPV and NPV were $77 \%, 89 \%, 89 \%$, and $77 \%$, respectively with $86 \%$ accuracy in the differentiation between the two pathogeneses of thrombocytopenia. A previous study involving 699 enrolled patients divided the causes of thrombocytopenia into two categories, patients with or without marrow diseases. In that study, the mean MPV measured using an MS-9 Automatic Full Digital Cell Counter in the marrow disease group was $7.3 \mathrm{fL}$ whereas that in the normal BM group was $8.62 \mathrm{fL}$, the difference being statistically significant. However, the sensitivity and specificity of MPV at a cut-off point of $8.15 \mathrm{fL}$ were only $67.7 \%$ and $65 \%$, respectively and a BM study for accurate diagnosis of the causes of thrombocytopenia was still recommended following these results ${ }^{12}$. The lower sensitivity and specificity of previous cut-off MPV values when compared to the current study might be explained by a difference between the populations included in the two studies. The previous study had a greater variety in underlying BM defects including megaloblastic anemia, MDS, and multiple myeloma (MM) comprising 31.2\%, 6.5\%, and $4.6 \%$ respectively in the underproductive BM group. Since megaloblastic anemia and MM had a relatively high MPV (mean 7.8 and $7.6 \mathrm{fL}$ ) compared with AA, AML, ALL (mean MPV of 6.4, 7.1, and 6.2, respectively), these might influence the sensitivity and specificity of cut-off points when comparing them to the current study which included no patients with mega-

loblastic anemia and MM. Moreover, the previous study included thrombocytopenic patients with anemia of chronic disease, totaling one-third $(31.9 \%)$ in the normal BM group. This disease ensures a relatively low MPV (mean 7.9 fL) when compared to "true" peripheral destruction causes of thrombocytopenia such as DIC, infections, and ITP (mean MPV of 10.1, 8.9, and 8.5 $\mathrm{fL}$, respectively) and might also affect the MPV cut-off value.

The difference between sensitivity and specificity of the MPV cut-off of $8.8 \mathrm{fL}$ in the training and validation cohorts in the current study might be also explained by the proportion of diseases included. This is bearing in mind that the sensitivity and specificity of this cut-off point were $60 \%$ and $50 \%$ in the training cohort but they increased to $77 \%$ and $89 \%$, respectively in the validation cohort, more reliable findings, because the BM studies were performed in all patients to confirm the causes of thrombocytopenia. Since ITP accounted for $100 \%$ of the over-destruction group in the validation cohort but only one-third in training cohort, it might affect the mean MPV in the over-destruction group in these cohorts (10.4 fL in validation set vs. $9.6 \mathrm{fL}$ in the training set) and consequently affected the sensitivity and specificity of this cutoff value.

The other factor that might affect the sensitivity and specificity of the MPV cutoff point is the degree of thrombocytopenia. A previous study reported a higher mean platelet count $\left(63.1 \times 10^{9} / \mathrm{L}\right)$ compared to all groups in both the training and validation sets and might lead to less change in MPV. ${ }^{6}$

The previous studies from Thailand

Table 2. Comparison of clinical characteristics between patients with underproductive bone marrow and those with over-destruction of platelets in the validation set.

Parameter $\begin{gathered}\text { Underproductive } \\ \text { bone marrow, } \\ \mathrm{N}=84(\%)\end{gathered}$
$\begin{gathered}\text { of platelets, } \\ \mathrm{N}=35(\%)\end{gathered}$

Gender

\begin{tabular}{lccc} 
Male & $56(66.7)$ & $22(62.9)$ & 0.69 \\
Female & $28(33.3)$ & $13(37.1)$ & \\
Age (years), Mean \pm SD & $43.5 \pm 18.4$ & $50.0 \pm 20.0$ & 0.84 \\
Diagnosis (\%) & AA 22 (26.2) & ITP, $35(100)$ & - \\
& AML 40 (47.6) & & \\
& ALL $12(14.3)$ & & \\
Hemoglobin $(\mathrm{g} / \mathrm{dL})$, Mean \pm SD & CMT $10(11.9)$ & & 0.03 \\
\hline White blood count $\left(\times 10^{9} / \mathrm{L}\right)$, Mean \pm SD & $8.4 \pm 1.8$ & $9.9 \pm 2.5$ & 0.03 \\
Platelet count $\left(\times 10^{9} / \mathrm{L}\right)$, Mean \pm SD & $2.6 \pm 36$ & $6.7 \pm 6.3$ & 0.45 \\
\hline Mean platelet volume $(\mathrm{fL})$, Mean \pm SD & $2.79 \pm 27.6$ & $41.1 \pm 26.6$ & 0.03 \\
\hline A, aplastic anemia; AML, acute myeloid leukemia (pre-chemotherapy); ALL, acute lymphoblastic leukemia (pre-chemotherapy); CMT,
\end{tabular}

AA, aplastic anemia; AML, acute myeloid leukemia (pre-chemotherapy); ALL, acute lymphoblastic leukemia (pre-chemotherapy); CMT, chemotherapy-induced myelosuppression; ITP, immune thrombocytopenia. 
used a mean MPV of the Thai population as $7.9 \mathrm{fL}^{13}$ to distinguish between hypodestructive and hyperdestructive thrombocytopenia cases which were confirmed with BM studies. A Coulter counter STKS was used to measure MPV. The result was prospectively evaluated and revealed a sensitivity, specificity, PPV, and NPV of $82.3 \%, 92.5 \%, 94.4 \%$, and $77.1 \%$, respectively. ${ }^{14}$ In that study, the mean MPV in the peripheral destruction group was $8.8 \mathrm{fL}$ compared to the $10.4 \mathrm{fL}$ found in the current study although ITP (both primary and secondary) accounted for the majority of patients, a statistic similar to this study. The variation of mean MPV in each study might be explained by the use of different automated blood analyzers. Standardized measurement of MPV may partly decrease this variation between studies in the future. Nevertheless, a mean MPV in cases of overdestruction of platelets was higher than that found in underproduction BM defects across all studies. ${ }^{7,12,14}$ Since there are variations in cut-off points as well as sensitivity and specificity in many studies, the MPV interpretation should be done together with careful history taking, and physical examination, which will lead to further appropriate investigations and management.

There were studies that used other automated platelet indices to discriminate between the causes of thrombocytopenia. The majority of them were differentiated from ITP. Firstly, the study by Kaito et al. used platelet distribution width (PDW) and platelet-to-large-cell ratio (P-LCR) as well as MPV to differentiate between thrombocytopenia resulting from AA and ITP. MPV was higher in patients with ITP compared to AA (mean $12.2 \mathrm{fL}$ vs. $10.2 \mathrm{fL}$, respectively, $\mathrm{P}<0.0001)$ in agreement with PDW and $\mathrm{P}$ LCR. ${ }^{9}$ Another two studies compared platelet indices between patients with ITP and hypoproliferative thrombocytopenia. The ITP group had a higher mean MPV (11.38 fL vs. $7.17 \mathrm{fL}$ in one study and $12.4 \mathrm{fL}$ vs. $9.7 \mathrm{fL}$ in another), ${ }^{10,11}$ as well as a higher PDW, and P-LCR. In one study, the cut-off values of 9-10 fL for MPV and 15-17 fL for PDW led to a sensitivity, specificity, PPV and NPV of $100 \%$ in the discrimination between two groups which were better than P-LCR ${ }^{10}$. An immature platelet fraction (IPF) is another platelet index that was studied in cases of childhood ITP. This index not only can discriminate thrombocytopenia from ITP and hematological malignancies under chemotherapy (median IPF $11.8 \%$ vs. $7 \%$ ) but can also distinguish acute and chronic ITP (median IPF 9.8\% vs. 19.4\%) as well as determine the active disease stage, response to treatment and relapsed disease.
Although MPV did not differentiate between acute and chronic ITP in that study, it still had value in discriminating between ITP and hematological malignancies (median MPV $11.6 \mathrm{fL} v s .10 .5 \mathrm{fL}$ ) as well as PDW, plateletcrit and P-LCR. ${ }^{15}$

The main strength of this study was the confirmation of a cut-off MPV value calculated from data collected from the training cohort in a validation cohort in which the causes of thrombocytopenia had been confirmed by BM studies in all patients. A limitation of the study was that the information from the training set was gathered from a review of medical records in which the BM investigation had only been completed in some patients. In addition, the training set was not well matched as regards age and sex as there were a predominance of females and younger patients in the underproductive $\mathrm{BM}$ group and this may well have impact on MPV. Another limitation was that no patients with MDS, MM or megaloblastic anemia were included in the cohort, as discussed previously, as well as diseases with sequestration of platelets such as hypersplenism and gestational thrombocytopenia. Nonetheless these diseases usually have clues from the patient history and physical examination and lead to specific investigations. ${ }^{16-18}$ This means the problem in the differentiation from hyperdestructive thrombocytopenia would be less. Some patients with diseases that are associated with both underproductive and overdestructive thrombocytopenia, for example chronic lymphocytic leukemia, were also not enrolled in this study but a BM study should be carried out in these cases. A final limitation was that patients with inherited thrombocytopenia were also not enrolled onto the study. A previous study showed an MPV at a cutoff value of $12.4 \mathrm{fL}$ had a high specificity (91\%) in distinguishing inherited macrothrombocytopenia from ITP. ${ }^{19}$ As a result, when using an MPV of more than 8.8 fL to indicate hyperdestructive thrombocytopenia, inherited macrothrombocytopenia is likely to be one exception and should be suspected if the MPV is very high. Future research into MPV in specific groups of patients that is more relevant in clinical practice such as patients with normal levels of hemoglobin, numbers of white blood cells, and no blasts is warranted.

In conclusion, MPV is useful as a screening test for differentiating the cause of thrombocytopenia. The value of MPV $\geq 8.8$ fL has acceptable sensitivity and specificity for the diagnosis of over-destructive thrombocytopenia and may help to avoid the need for invasive bone marrow examination in these patients.

\section{References}

1. Stasi R. How to approach thrombocytopenia. Hematol Am Soc Hematol Educ Program 2012;2012:191-7.

2. Neunert C, Lim W, Crowther M, et al. The American Society of Hematology 2011 evidence-based practice guideline for immune thrombocytopenia. Blood 2011;117:4190-207.

3. Wada H, Thachil J, Di Nisio M, et al. Guidance for diagnosis and treatment of disseminated intravascular coagulation from harmonization of the recommendations from three guidelines. J Thromb Haemost 2013;11:761-7.

4. Leader A, Pereg D, Lishner M. Are platelet volume indices of clinical use? A multidisciplinary review. Ann Med 2012;44:805-16.

5. Bessman JD, Gilmer PR, Gardner FH. Use of mean platelet volume improves detection of platelet disorders. Blood Cell 1985;11:127-35.

6. Levin J, Bessman JD. The inverse relation between platelet volume and platelet number. Abnormalities in hematologic disease and evidence that platelet size does not correlate with platelet age. J Lab Clin Med 1983;101:295-307.

7. Bowles KM, Cooke LJ, Richards EM, Baglin TP. Platelet size has diagnostic predictive value in patients with thrombocytopenia. Clin Lab Haem 2005;27:370-3.

8. Abe Y, Wada H, Tomatsu H, et al. A simple technique to determine thrombopoiesis level using immature platelet fraction (IPF). Thromb Res 2006;118:463-9.

9. Kaito K, Otsubo H, Usui N. Platelet size deviation width, platelet large cell ratio, and mean platelet volume have sufficient sensitivity and specificity in the diagnosis of immune thrombocytopenia. Br J Haematol 2005;128:698-702.

10. Ntaios G, Papadopoulos A, Chatzinikolaou A. Increased values of mean platelet volume and platelet size deviation width may provide a safe positive diagnosis of idiopathic thrombocytopenic purpura. Acta Haematol 2008;119:173-7.

11. Negash M, Tsegaye A, Medhin AG. Diagnostic predictive value of platelet indices for discriminating hypo productive versus immune thrombocytopenia purpura in patients attending a tertiary care teaching hospital in Addis Ababa, Ethiopia. BMC Hematology 2016;16:18.

12. Chandra H, Chandra S, Rawat A, Verma 
SK. Role of mean platelet volume as discriminating guide for bone marrow disease in patients with thrombocytopenia. Int J Lab Hematol 2010;32:498505.

13. Pathepchotiwong K., Dhareruchta P, Adirojananon W. Platelet parameter in healthy subjects analyzed by automation analyzer. Thai J Hematol Transf Med 2001;11:93-100.

14. Numbenjapon T, Mahapo N, Pornvipawee R, et al. A prospective evaluation of normal mean platelet volume in discriminating hyperdestructive thrombocytopenia from hypoproductive thrombocytopenia. Int $\mathrm{J}$ Lab Hem 2008;30:408-14.

15. Adly AAM, Ragab IA, Ismail EAR, Farahat MM. Evaluation of the immature platelet fraction in the diagnosis and prognosis of childhood immune thrombocytopenia. Platelets 2015;26:645-50.

16. Fenaux P, Haase D, Sanz GF, et al. Myelodysplastic syndromes: ESMO Clinical Practice Guidelines for diagnosis, treatment and follow-up. Ann Oncol 2014;25:iii57-69.

17. Rajkumar SV, Dimopoulos MA, Palumbo A, et al. International
Myeloma Working Group updated criteria for the diagnosis of multiple myeloma. Lancet Oncol 2014;15:e53848.

18. Green R, Datta Mitra A. Megaloblastic Anemias: Nutritional and Other Causes. Med Clin North Am 2017;101:297-317.

19. Noris P, Klersy C, Gresele P, et al. Platelet size for distinguishing between inherited thrombocytopenias and immune thrombocytopenia: a multicentric, real life study. $\mathrm{Br} \mathrm{J}$ Haematol 2013;162:112-9. 